\title{
Salivary microbial changes during the first 6 months of orthodontic treatment
}

\author{
Mei Zhao ${ }^{1}$, Min Liu ${ }^{1}$, Wei Chen ${ }^{1}$, Haiping Zhang ${ }^{2}$, Yuxing Bai ${ }^{2}$, Wen Ren ${ }^{\text {Corresp. } 1}$ \\ ${ }^{1}$ Department of Preventive Dentistry, School of Stomatology, Capital Medical University, Beijing, China \\ 2 Department of Orthodontics, School of Stomatology, Capital Medical University, Beijing, China \\ Corresponding Author: Wen Ren \\ Email address: renwen90@foxmail.com
}

Background. Orthodontic treatment is widely used to treat malocclusion. However, the influence of treatment on the oral microbiome remains unclear. In this study, we investigated salivary microbial changes in patients undergoing orthodontic treatment.

Methods. In total, 19 orthodontic patients participated in this study. Saliva samples were collected at the following three timepoints: before (T0) and 3 months (T1) and 6 months (T2) after the placement of orthodontic appliances. High-throughput sequencing was performed based on the 16S rRNA gene V4 region.

Results. The phyla of Proteobacteria, Bacteroidetes, Firmicutes, Actinobacteria and Fusobacteria were predominant. Observed Species, Chaol and ACE, which represent $\alpha$ diversity, were significantly decreased at T1 and subsequently increased at T2. In addition, the $\beta$ diversity at T1 based on the BrayCurtis distances differed from T0 and T2. The relative abundances of Prevotella, Porphyromonas and Peptostreptococcus were decreased with treatment, whereas those of Capnocytophaga and Neisseria exhibited the opposite results. In total, 385 of 410 operational taxonomic units were shared at T0, T1 and T2. The co-occurrence networks with hub nodes at T1 were the most complex.

Conclusion. Orthodontic treatment temporarily affected the saliva microbial community. This dynamic alteration in species did not induce deterioration in oral health. Oral hygiene instructions were necessary and should be emphasized during each visit. Further studies with longer observation periods and more participants are required. 


\section{Salivary microbial changes during the first 6 months of 2 orthodontic treatment}

3 Mei Zhao ${ }^{1}$, Min Liu ${ }^{1}$, Wei Chen ${ }^{1}$, Haiping Zhang ${ }^{2}$, Yuxing Bai ${ }^{2}$, Wen Ren ${ }^{1}$

$5{ }^{1}$ Department of Preventive Dentistry, School of Stomatology, Capital Medical University,

6 Beijing, China

$7{ }^{2}$ Department of Orthodontics, School of Stomatology, Capital Medical University, Beijing,

8 China

10 Corresponding author:

11 Wen Ren ${ }^{1}$

12 Tian Tan Xi Li No. 4, Dongcheng District, Beijing, 100050, China

13 Email address: renwen90@,foxmail.com

15 This manuscript was edited to ensure proper English language, grammar, punctuation, spelling,

16 and overall style by one or more highly qualified native English-speaking editors at AJE (EC4F-

$17 \quad 320 \mathrm{~F}-3326-311 \mathrm{~B}-2 \mathrm{C} 53)$. 
18 Abstract

19

Background. Orthodontic treatment is widely used to treat malocclusion. However, the influence of treatment on the oral microbiome remains unclear. In this study, we investigated salivary microbial changes in patients undergoing orthodontic treatment.

Methods. In total, 19 orthodontic patients participated in this study. Saliva samples were collected at the following three timepoints: before (T0) and 3 months (T1) and 6 months (T2) after the placement of orthodontic appliances. High-throughput sequencing was performed based on the 16S rRNA gene V4 region.

Results. The phyla of Proteobacteria, Bacteroidetes, Firmicutes, Actinobacteria and Fusobacteria were predominant. Observed Species, Chao1 and ACE, which represent $\alpha$ diversity, were significantly decreased at T1 and subsequently increased at T2. In addition, the $\beta$ diversity at T1 based on the Bray-Curtis distances differed from T0 and T2. The relative abundances of Prevotella, Porphyromonas and Peptostreptococcus were decreased with treatment, whereas those of Capnocytophaga and Neisseria exhibited the opposite results. In total, 385 of 410 operational taxonomic units were shared at T0, T1 and T2. The co-occurrence networks with hub nodes at T1 were the most complex.

Conclusion. Orthodontic treatment temporarily affected the saliva microbial community. This dynamic alteration in species did not induce deterioration in oral health. Oral hygiene instructions were necessary and should be emphasized during each visit. Further studies with longer observation periods and more participants are required.

Keywords: Orthodontic treatment, Microbiome, 16S rRNA gene high-throughput sequencing 


\section{Introduction}

40 Orthodontic treatment is an effective method for correcting malocclusion, which is a common

41 disease in the oral and maxillofacial region. The increased accumulation of dental plaque with the placement of orthodontic appliances induces the occurrence of dental diseases (Lee et al. 2005; van Gastel et al. 2008). Dental plaque is the primary etiological factor of white spot lesions, caries, gingivitis and periodontitis, which represent complications of orthodontic treatment and pose a great threat to oral health (Al-Anezi 2014). In some cases, severe complexes decreased at 3 months (Bergamo et al. 2019).

Peer) reviewing PDF | (2020:07:51390:1:0:CHECK 18 Oct 2020) 
60

61

62

63

64

65

66

67

Many reports have evaluated orthodontic treatment from a microbiological aspect using traditional methods, such as culture, DNA-DNA hybridization, and polymerase chain reaction.

The oral cavity harbors more than 700 species; of these species, $35 \%$ cannot be cultivated under current experimental conditions (Dewhirst et al. 2010). High-throughput sequencing overcomes the limitations of traditional methods, providing a more comprehensive perspective of the global microbial community. This technique has been widely used in gut and dental research

(Citronberg et al. 2018; Serena et al. 2018; Teng et al. 2015; Xu et al. 2015). In this study, we examined a longitudinal cohort of orthodontic patients over a 6-month period. Salivary microbial communities were characterized and compared to better understand the microbial changes occurring during the early stage of orthodontic treatment.

\section{Materials and Methods}

\section{Ethics Statement}

This study was approved by the Ethics Committee of Capital Medical University School of Stomatology (CMUSH-IRB-KJ-PJ-2017-04). Written informed consent was obtained from all the participants or their guardians.

\section{Study Design and Sample Collection}

In total, 70 participants from the Department of Orthodontics underwent an oral examination.

Nineteen of these patients underwent a six-month follow-up and were recruited for this study.

During the follow-up, the following three timepoints were selected: before the start of the 
orthodontic treatment (T0) and 3 months (T1) and 6 months (T2) after the treatment. None of the participants had severe periodontal diseases, colds, or systemic diseases or received antimicrobial or anti-inflammatory therapy within 6 months prior to the study. Saliva samples were collected by a saliva collection kit (Zeesan Biotech Co., Xiamen, China). The patients were not allowed to eat, drink, smoke or chew gum for 30 minutes before giving their samples. In total, $2 \mathrm{ml}$ of unstimulated saliva was collected, and Saliva Stabilization Solution was added to the samples. All samples were stored at $-80^{\circ} \mathrm{C}$ until DNA extraction. The Quigley-Hein Plaque Index was selected and evaluated by one experienced dentist. Oral hygiene instructions were provided during each appointment.

DNA Extraction, PCR Amplification and High-throughput Sequencing Genomic DNA was extracted using prepIT · L2P (DNA Genotek, Canada) according to the manufacturer's instructions. The DNA purity was determined using a NanoDrop 8000 Spectrophotometer (Thermo, USA). The hypervariable region V4 of the bacterial 16S rRNA gene was amplified via PCR using the primers 515F (5'-GTGCCAGCMGCCGCGGTAA-3') and 806R (5'-GGACTACHVGGGTWTCTAAT-3'). The PCR amplicons were sequenced using the Illumina HiSeq platform with a pair-end 250-bp strategy at the BGI Institute (Shenzhen, China). The sequences were submitted to the NCBI Sequence Read Archive under accession number PRJNA648248. 
102 The 16S rRNA gene sequences were processed using the QIIME pipeline (ver. 1.9.1). The 103 sequencing data were demultiplexed using a unique barcode assigned to each sample. The 104 sequences were trimmed when the average quality score over a 25-bp sliding window was less 105 106 107 108 
123 of the participants was $21.1 \pm 7.4$ years; the Friedman test and $\chi^{2}$ analysis revealed no significant

124 differences in terms of plaque index or gender (Table 1). The bacterial community profile in each

125 sample was determined by $16 \mathrm{~S}$ rRNA gene amplification targeting the V4 region using the

126 Illumina HiSeq platform. After processing, we obtained a total of 4,075,131 clean reads, ranging

127 from 60,518 to 90,158 per sample (Table 1). Good's coverage was greater than $99.9 \%$ in all the

128 samples. Good's coverage and the rarefaction curves indicated that the sequencing coverage was

129 adequate under the chosen depth (Table 1 \& Fig. S1). These sequences were clustered into 410

130 OTUs using a 3\% dissimilarity cut-off.

131

132

Microbial Diversity in the Microbial Community

133 The indices Observed Species, Chaol and ACE representing microbial richness were calculated

134 from 60,518 reads. These three indices were significantly decreased at $\mathrm{T} 1$ and increased at $\mathrm{T} 2$

135 (Fig. 1a). To investigate the microbial structure, we calculated the $\beta$ diversity based on the Bray-

136 Curtis distance and visualized the microbial structure by principle coordinate analysis (PCoA)

137 plots. The analysis revealed that the T1 samples tended to cluster together and were well

138 separated from the T0 and T2 samples (Fig. 1b).

139

140

OTUs and Taxa with Different Abundances

141 In total, 13 phyla, 21 classes, 32 orders, 55 families, and 78 genera were observed in this study.

142 Five phyla, Proteobacteria, Bacteroidetes, Firmicutes, Actinobacteria and Fusobacteria, were

143 predominant in all three groups, accounting for more than $99 \%$ of all bacteria. To further explore

PeerJ reviewing PDF | (2020:07:51390:1:0:CHECK 18 Oct 2020) 
144 the changes in specific taxa throughout the first six months of orthodontic treatment, we 145 compared the relative abundance of the salivary microbiota (Fig. 2, Fig. S2). The abundance of 146 Prevotella, including OTU368, OTU118, OTU085, OTU277, and OTU325, decreased as the 147 treatment progressed (Fig. 2a, Fig. S2e). Porphyromonas (OTU034), which is a genus of Bacteroidetes, and Peptostreptococcus (OTU253) exhibited similar results, whereas Capnocytophaga (OTU047) and Neisseria (OTU305) exhibited the opposite results (Fig. a, b, c, Fig. S2e).

\section{Core Microbiome and Co-occurrence Networks}

Despite the OTUs of different relative abundances, 385 of the 410 OTUs were shared among all

three groups (Fig. 3). The remaining 25 OTUs are also shown in Fig. 4, and the relative

abundances of these OTUs were less than $0.01 \%$ (Table S1-1). The core microbiome was defined as the OTUs present in all 57 samples in this study. In total, 26 OTUs were retained. Neisseria (OTU278), Prevotella (OTU368) and Streptococcus (OTU192) predominated among these 26 OTUs, with relative abundances of $9.47 \%, 9.34 \%$ and $6.82 \%$, respectively (Table S1-2). calculating and visualizing the Spearman's correlation coefficients for all OTUs in the T0 group, we initially obtained a network of 393 OTUs (nodes) with 4,611 linkages (edges). Nodes with less than 15 edges were removed. The remaining 30 nodes were identified as hub nodes, with 408 edges (289 positive edges and 119 negative edges, Fig. 4a). Similarly, the networks of the T1 and T2 groups were generated and had no negative edges (Fig. 4b, c). Of all three networks, 
165 the T1 network exhibited the most linkages $(\mathrm{p}<0.05)$. Prevotella and Leptotrichia were the most 166 prevalent genera (Table S2).

167

168

\section{Discussion}

169 Increasing numbers of people choose to receive orthodontic treatment. The complications of orthodontic treatment are worthy of attention. Previous studies have indicated that the placement of orthodontic appliances increased the risk of dental caries and periodontal disease, which are the most common diseases of the oral cavity. The etiology and progression of caries or periodontitis can be better understood by focusing on the consortia of organisms rather than one or several species (Xu et al. 2015). Thus, in this study, we used 16S rRNA gene high-throughput sequencing to explore the microbial changes in orthodontic patients to provide a general view of the bacterial community. Consistent with previous studies, the phyla Proteobacteria,

Bacteroidetes, Firmicutes, Actinobacteria and Fusobacteria were the most abundant (Wang et al. 2019). Our results indicate that the microbial richness was reduced at $\mathrm{T} 1$ and increased at $\mathrm{T} 2$

(Fig. 1a). In addition, the community structure at T1 clustered apart from that at T0 and T2 (Fig.

1b). However, the cross-sectional studies conducted by Sun et al. and Wang et al. suggest an increased Shannon index in orthodontic patients compared with that in controls, indicating greater microbial diversity (Sun et al. 2018; Wang et al. 2019). Sample sources, ages, eating habits, frequency of brushing teeth, and type of appliances are all factors that can affect the results (Dogramaci et al. 2020). Synergistic, mutualistic and antagonistic effects among microorganisms contribute to the development of polymicrobial interactions. Given these 
186

187

complex interactions, we generated co-occurrence networks at T0, T1 and T2 and selected hub nodes (Fig. 4). The networks at T1 differed from those at T0 and T2, with the most linkages, indicating that the microbial community structure is temporary and not permanent.

Neisseria is an early colonizer of the tooth surface, and some studies have indicated that Neisseria is associated with improved oral health or reduced gingivitis (Lif Holgerson et al. 2020). Previous studies reported that the abundance of Neisseria was reduced over time following treatment with fixed appliances (Koopman et al. 2015; Wang et al. 2019). In contrast, our study revealed the opposite result (Fig. 2b). The oral health conditions were potentially improved because we provided regular oral hygiene instructions before the initiation of treatment and reinforced this information during each visit. Prevotella spp. are gram-negative, nonmotile and rod-shaped bacteria that thrive under anaerobic growth conditions (Moller et al. 2020). Prevotella spp. can colonize the human mouth and are considered host-associated bacteria. Several Prevotella species are opportunistic pathogens and known for their role in periodontal disease ( $\mathrm{Li}$ et al. 2020). One systematic review concluded that the subgingival pathogen levels exhibited temporary increases after orthodontic appliance placement and that these levels returned to the pretreatment levels after several months (Guo et al. 2017). This finding indicates that orthodontic treatment might not permanently induce periodontal disease by affecting the subgingival periodontal pathogen level. In contrast, in this study, the relative abundance of Prevotella was decreased throughout the first 6 months of orthodontic treatment (Fig. 2a). Other studies have reported similar results (Wang et al. 2019). Bergamo et al. reported that Prevotella nigrescens was present at high levels before bonding using checkerboard DNA-DNA 
207 hybridization and that these levels were decreased 60 and 90 days after orthodontic appliance 208 placement (Bergamo et al. 2019). However, not all Prevotella species exhibited the same

209

210

211

212 changes. The Prevotella intermedia levels exhibited no significant differences during the observation period (Bergamo et al. 2019; Guo et al. 2019). Another important role of Prevotella spp. involves providing key nutrients to Peptostreptococcus sp. (James 2010). The fact that the relative abundance of Peptostreptococcus (OTU253) exhibited a trend similar to that of Prevotella provides evidence supporting this important role (Fig. 2c).

Enamel demineralization is caused by the effects of acid products on the carbohydrate metabolism of bacterial species. These species grow 6 or 12 weeks after orthodontic appliance bonding (Sanpei et al. 2010). Demineralization can cause white spot lesions and may result in dental caries during orthodontic treatment if not treated. S. mutans, Streptococcus sobrinus and Lactobacillus spp. are the main causative microorganisms of enamel demineralization. Many studies have reported changes in these species using different methods at different timepoints. For example, through the traditional culture method, significantly higher Lactobacillus CFU counts were found at 2-month follow-up following fixed orthodontic treatment (Kupietzky et al. 2005). A significant increase in Streptococcus spp. was noted only 1 week after the start of therapy in Reichardt's study using matrix-assisted laser desorption/ionization time-of-flight mass spectrometry (Reichardt et al. 2019). Jing et al. reported that the S. mutans abundance remained stable during the first 6 months and was significantly increased at 18 months; Lactobacillus abundance exhibited a slight but nonsignificant increase, as demonstrated by qPCR (Jing et al. 
227 2019). Using Dentocult SM and Dentocult LB, Maret et al. found that wearing a fixed

228 orthodontic appliance was associated with high levels of S. mutans and Lactobacillus spp. (Maret

229 et al. 2014). However, in our study, the relative abundance and prevalence of $S$. mutans, $S$.

230 sobrinus and Lactobacillus spp. did not obviously change before and after 6 months of treatment

231 based on the 16S rRNA gene high-throughput sequencing results. Thus, determining which

232 method is more reliable and the time point at which orthodontic treatment can influence the

233 progression of microbial changes is worthy of further investigation. Notably, Neisseria

234 (OTU278), Prevotella (OTU368) and Streptococcus (OTU192) were the predominant genera in

235 the core microbiome (Table S1-2).

236 This study suggests that alterations occur in the oral microbiota following orthodontic

treatment. The orthodontic treatment temporarily affected the saliva microbial diversity. As the

relative abundances of $S$. mutans and Lactobacillus spp. showed no significant differences,

orthodontic treatment does not induce deterioration in oral health from this perspective. Oral

hygiene instructions were necessary and should be emphasized during each visit.

241 One limitation of this study is that some aspects of malocclusion, such as crowing and

crossbite, were not considered. Given the relatively small sample size and short observation time,

243 it is necessary to be aware that our research is a preliminary investigation of the salivary

244 microbial community in orthodontic patients.

\section{Conclusions}

247 In this study, we found that the placement of orthodontic appliances may have a temporary 
248 impact on the salivary microflora. The dynamic alteration in species and orthodontic treatment

249 did not induce deterioration of oral health. Further studies of high methodological quality

250 involving more participants and longer-term observations are required to provide more reliable

251 evidence regarding this issue.

252

253 Funding

254 This work was supported by a grant from the Beijing Municipal Administration of Hospitals

255 Clinical Medicine Development of Special Funding Support (ZYLX201703).

256

257

258

259

260

261

262

263

264 inflammation. ISME J 7:1016-1025. 10.1038/ismej.2012.174

Al-Anezi SA. 2014. Dental plaque associated with self-ligating brackets during the initial phase of orthodontic treatment: A 3-month preliminary study. J Orthod Sci 3:7-11. 10.4103/2278-0203.127550

Bergamo AZN, de Oliveira KMH, Matsumoto MAN, Nascimento CD, Romano FL, da Silva RAB, da Silva LAB, and Nelson-Filho P. 2019. Orthodontic appliances did not increase risk of dental caries and periodontal disease under preventive protocol. Angle Orthodontist 89:25-32. 10.2319/022118-139.1

Citronberg JS, Curtis KR, White E, Newcomb PA, Newton K, Atkinson C, Song X, Lampe JW, and Hullar MA. 2018. Association of gut microbial communities with plasma lipopolysaccharide-binding protein (LBP) in premenopausal women. ISME J 12:1631-1641. 10.1038/s41396-018-0064-6 
Dewhirst FE, Chen T, Izard J, Paster BJ, Tanner AC, Yu WH, Lakshmanan A, and Wade WG. 2010. The human oral microbiome. Journal of Bacteriology 192:5002-5017. 10.1128/JB.00542-10

Dogramaci EJ, Naini FB, and Brennan DS. 2020. The long-term influence of orthodontic treatment on dental knowledge and behaviour: An Australian cohort study. Journal of Dentistry:103345. 10.1016/j.jdent.2020.103345

Guo R, Lin Y, Zheng Y, and Li W. 2017. The microbial changes in subgingival plaques of orthodontic patients: a systematic review and meta-analysis of clinical trials. BMC Oral Health 17:90. 10.1186/s12903-0170378-1

Guo R, Liu H, Li X, Yang Q, Jia L, Zheng Y, and Li W. 2019. Subgingival Microbial Changes During the First 3 Months of Fixed Appliance Treatment in Female Adult Patients. Current Microbiology 76:213-221. $10.1007 / \mathrm{s} 00284-018-1610-1$

James FY. 2010. Understanding corn and wheat: Oxford: Oxford University Press.

Jing D, Hao J, Shen Y, Tang G, Lei L, and Zhao Z. 2019. Effect of fixed orthodontic treatment on oral microbiota and salivary proteins. Experimental and Therapeutic Medicine 17:4237-4243. 10.3892/etm.2019.7401

Koopman JE, van der Kaaij NC, Buijs MJ, Elyassi Y, van der Veen MH, Crielaard W, Ten Cate JM, and Zaura E. 2015. The Effect of Fixed Orthodontic Appliances and Fluoride Mouthwash on the Oral Microbiome of Adolescents - A Randomized Controlled Clinical Trial. PloS One 10:e137318. 10.1371/journal.pone.0137318

Kupietzky A, Majumdar AK, Shey Z, Binder R, and Matheson PB. 2005. Colony forming unit levels of salivary Lactobacilli and Streptococcus mutans in orthodontic patients. Journal of Clinical Pediatric Dentistry 30:51-53. 
290

291

292

293

294

295

296

297

298

299

300

301

302

303

304

305

306

307

308

309

310
Lee SM, Yoo SY, Kim HS, Kim KW, Yoon YJ, Lim SH, Shin HY, and Kook JK. 2005. Prevalence of putative periodontopathogens in subgingival dental plaques from gingivitis lesions in Korean orthodontic patients. Journal of Microbiology 43:260-265.

Li Y, Tan X, Zhao X, Xu Z, Dai W, Duan W, Huang S, Zhang E, Liu J, Zhang S, Yin R, Shi X, Lu Z, and Pan Y. 2020. Composition and function of oral microbiota between gingival squamous cell carcinoma and periodontitis. Oral Oncology 107:104710. 10.1016/j.oraloncology.2020.104710

Lif Holgerson P, Esberg A, Sjodin A, West CE, and Johansson I. 2020. A longitudinal study of the development of the saliva microbiome in infants 2 days to 5 years compared to the microbiome in adolescents. Scientific Reports 10:9629. 10.1038/s41598-020-66658-7

Maret D, Marchal-Sixou C, Vergnes JN, Hamel O, Georgelin-Gurgel M, Van Der Sluis L, and Sixou M. 2014. Effect of fixed orthodontic appliances on salivary microbial parameters at 6 months: a controlled observational study. J Appl Oral Sci 22:38-43. 10.1590/1678-775720130318

Moller B, Kollert F, Sculean A, and Villiger PM. 2020. Infectious Triggers in Periodontitis and the Gut in Rheumatoid Arthritis (RA): A Complex Story About Association and Causality. Frontiers in Immunology 11:1108. 10.3389/fimmu.2020.01108

Reichardt E, Geraci J, Sachse S, Rodel J, Pfister W, Loffler B, Wagner Y, Eigenthaler M, and Wolf M. 2019. Qualitative and quantitative changes in the oral bacterial flora occur shortly after implementation of fixed orthodontic appliances. American Journal of Orthodontics and Dentofacial Orthopedics 156:735744. 10.1016/j.ajodo.2018.12.018

Sanpei S, Endo T, and Shimooka S. 2010. Caries risk factors in children under treatment with sectional brackets. Angle Orthodontist 80:509-514. 10.2319/072909-431.1

Peer) reviewing PDF | (2020:07:51390:1:0:CHECK 18 Oct 2020) 
311 Serena C, Ceperuelo-Mallafre V, Keiran N, Queipo-Ortuno MI, Bernal R, Gomez-Huelgas R, Urpi-Sarda M,

Sabater M, Perez-Brocal V, Andres-Lacueva C, Moya A, Tinahones FJ, Fernandez-Real JM, Vendrell J, and Fernandez-Veledo S. 2018. Elevated circulating levels of succinate in human obesity are linked to specific gut microbiota. ISME J 12:1642-1657. 10.1038/s41396-018-0068-2

Sun F, Ahmed A, Wang L, Dong M, and Niu W. 2018. Comparison of oral microbiota in orthodontic patients and healthy individuals. Microbial Pathogenesis 123:473-477. 10.1016/j.micpath.2018.08.011

Teng F, Yang F, Huang S, Bo C, Xu ZZ, Amir A, Knight R, Ling J, and Xu J. 2015. Prediction of Early Childhood Caries via Spatial-Temporal Variations of Oral Microbiota. Cell Host Microbe 18:296-306. 10.1016/j.chom.2015.08.005

van Gastel J, Quirynen M, Teughels W, Coucke W, and Carels C. 2008. Longitudinal changes in microbiology and clinical periodontal variables after placement of fixed orthodontic appliances. Journal of Periodontology 79:2078-2086. 10.1902/jop.2008.080153

Wang Q, Ma JB, Wang B, Zhang X, Yin YL, and Bai H. 2019. Alterations of the oral microbiome in patients treated with the Invisalign system or with fixed appliances. American Journal of Orthodontics and Dentofacial Orthopedics 156:633-640. 10.1016/j.ajodo.2018.11.017

Xu H, Hao W, Zhou Q, Wang W, Xia Z, Liu C, Chen X, Qin M, and Chen F. 2014. Plaque bacterial microbiome diversity in children younger than 30 months with or without caries prior to eruption of second primary molars. PloS One 9. 10.1371/journal.pone.0089269

Xu X, He JZ, Xue J, Wang Y, Li K, Zhang KK, Guo Q, Liu XH, Zhou Y, Cheng L, Li MY, Li YQ, Li Y, Shi WY, and Zhou XD. 2015. Oral cavity contains distinct niches with dynamic microbial communities. Environmental Microbiology 17:699-710. 


\section{Figure Legends}

333

334

335

336

337

338

339

340

341

342

343

344

345

346

347

348

349

350

351

Figure 1 Comparison of the microbial diversity at T0, T1 and T2. (a) Sequences $(60,518)$

were randomly subsampled to obtain equal numbers of sequences from each dataset. Indices,

namely, Observed Species, Chao1 and ACE, representing $\alpha$ diversity are shown. (b)

Phylogenetic distances between samples were calculated via the Bray-Curtis distance. Each dot in the scatter plot represents one sample. The percentage of variation is indicated on the $\mathrm{x}$ and $\mathrm{y}$ axes. T1 samples significantly differed from the T0 and T2 samples (ANOSIM, $\mathrm{p}<0.05$ ).

Figure 2 Taxa with different relative abundances at the OTU level. Bars represent the mean relative abundance $( \pm \mathrm{SD}) .{ }^{*} \mathrm{p}<0.05$, Friedman test.

Figure 3 Shared and unique OTUs at T0, T1 and T2. Venn diagram showing the size of each oral bacterial community. In total, 385 OTUs were shared by all three groups. OTUs in the box are unique OTUs.

Figure 4 Co-occurrence networks of hub nodes. Spearman's correlation coefficients (SCCs) were calculated between different OTUs. Each node represents one OTU. The edge between two nodes represents a significant correlation $(|\mathrm{SCC}| \geq 0.6, \mathrm{p}<0.05)$. Red and gray edges indicate positive and negative correlations, respectively. 
Figure 1

Comparison of microbial diversity of $\mathrm{T} 0, \mathrm{~T} 1$ and $\mathrm{T} 2$.

(a) Sequences $(60,518)$ were randomly subsampled to obtain equal numbers of sequences from each dataset. Indices, namely, Observed Species, Chaol and ACE, representing $\alpha$ diversity are shown. (b) Phylogenetic distances between samples were calculated via BrayCurtis distance. Each dot in the scatter plot represents one sample. The percentage of variation is indicated on the $x$ and $y$-axes. T1 significantly differed from T0 and T2 (ANOSIM, $p$ $<0.05)$.

a

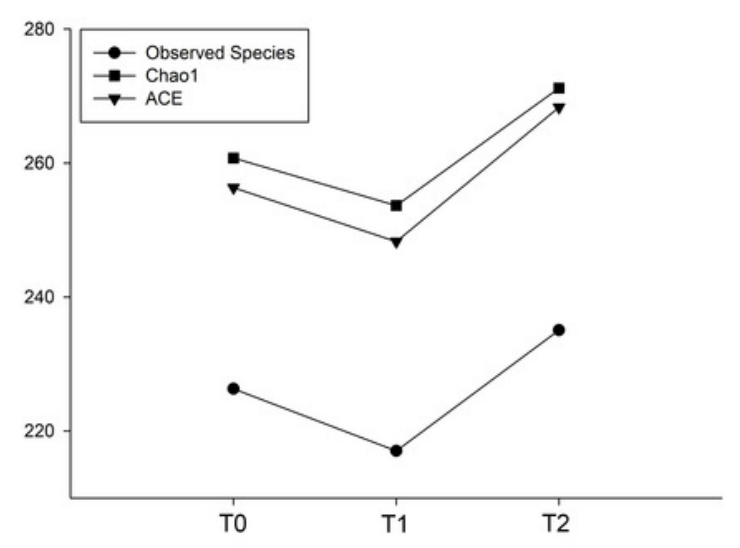

b

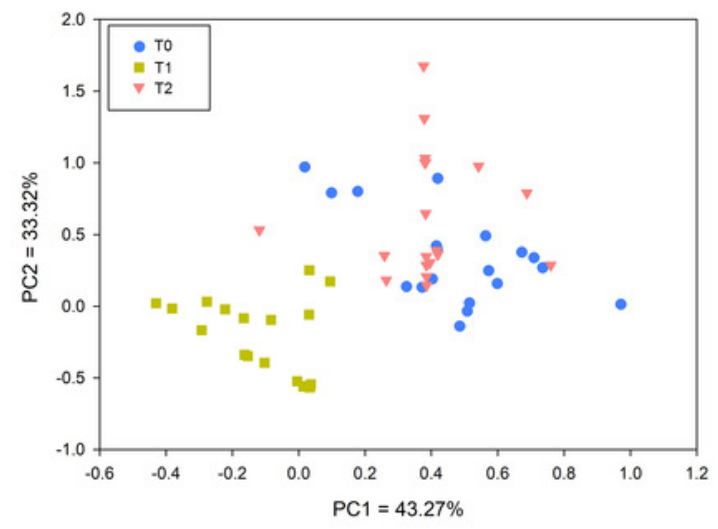


Figure 2

Taxa with different relative abundances at the OTU level.

Bars represent the mean relative abundance $( \pm S D) .{ }^{*} p<0.05$, Friedman test. 
a

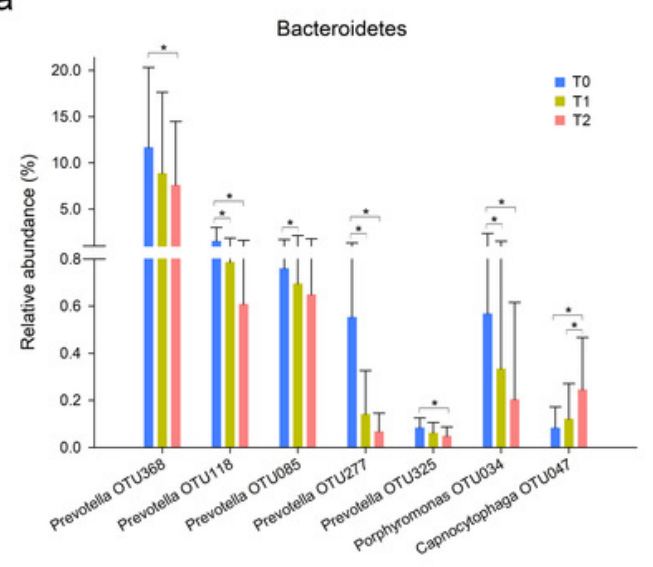

C

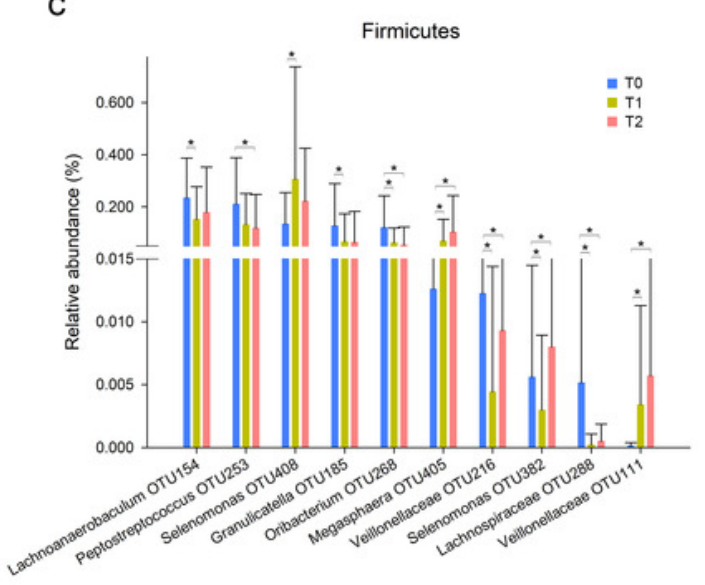

e

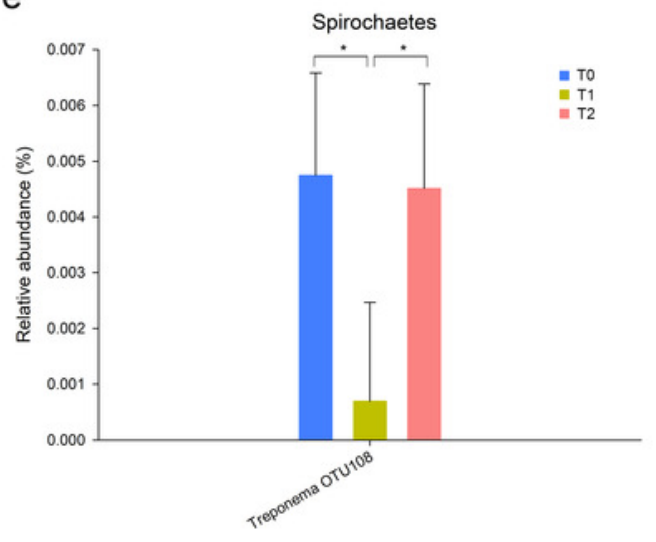

b

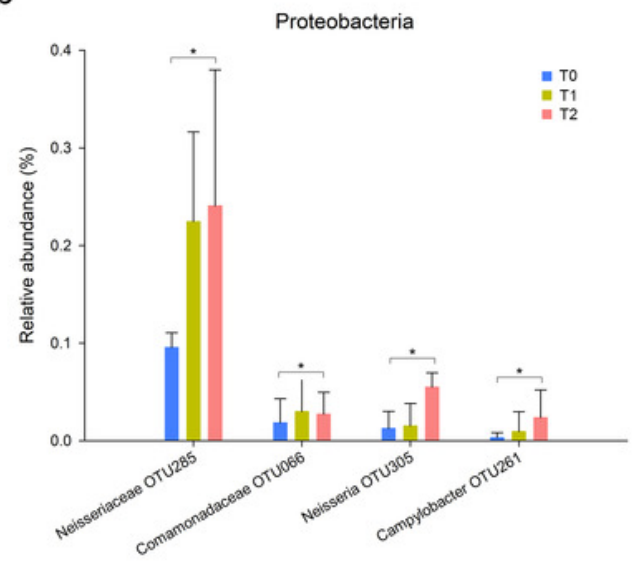

d

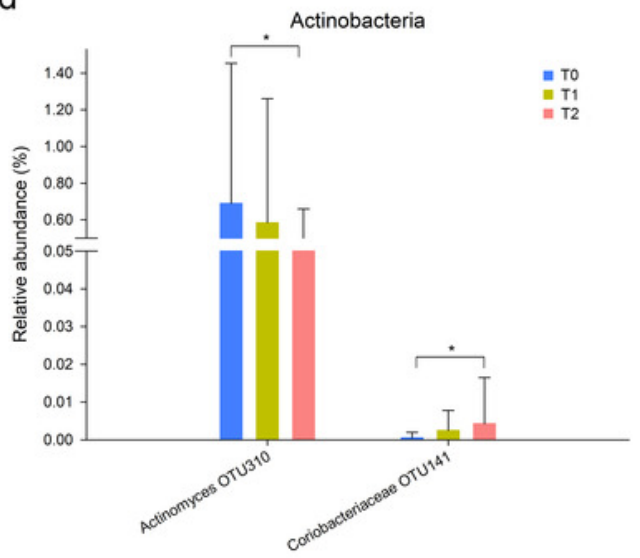

f

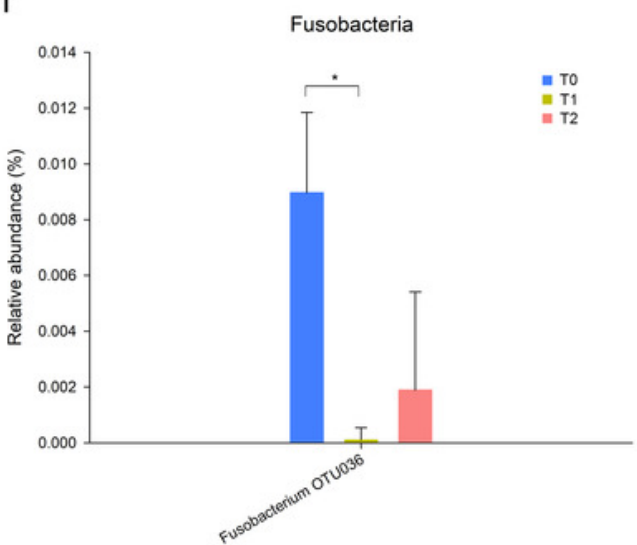


Figure 3

Shared and unique OTUs of T0, T1 and T2.

The Venn diagram shows the number of each oral bacterial community. A total of 385 OTUs were shared by all the three groups. OTUs in the box were unique OTUs. 
Figure 4

Co-occurrence networks of hub nodes.

Spearman's correlation coefficients (SCC) were calculated between different OTUs. Each node represents one OTU. The edge between two nodes represents significant correlations $(|S C C| \geq 0.6, p<0.05)$. Red and gray edges indicate positive and negative correlations, respectively.

a

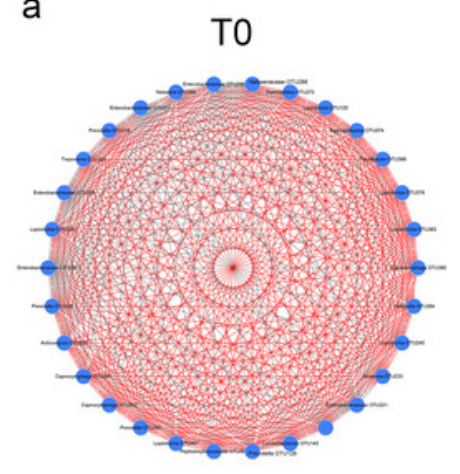

b

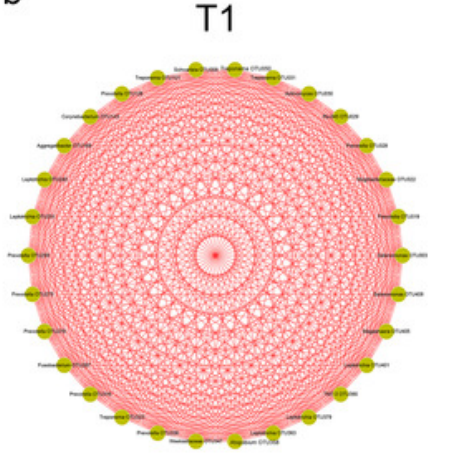

C

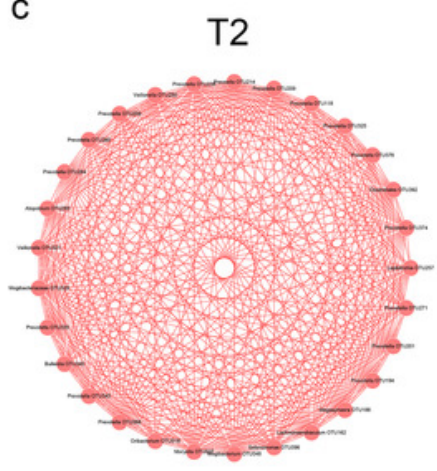


Table $\mathbf{1}$ (on next page)

Basic Information 
Table 1 Basic Information

\begin{tabular}{cccccc}
\hline$\square$ & plaque index & Raw Reads & Clean Reads & OTU & Good's coverage (\%) \\
\hline T0 & $2.37 \pm 0.40$ & $78785 \pm 15280$ & $75167 \pm 13922$ & $239 \pm 40$ & $99.94 \pm 0.01$ \\
T1 & $1.84 \pm 0.30$ & $74011 \pm 13588$ & $69250 \pm 7047$ & $211 \pm 57$ & $99.93 \pm 0.02$ \\
T2 & $1.79 \pm 0.32$ & $72738 \pm 7396$ & $70064 \pm 6777$ & $229 \pm 27$ & $99.93 \pm 0.02$ \\
\hline
\end{tabular}

1 